\title{
Médiévales
}

Langues, Textes, Histoire

78 | printemps 2020

Moyen Âge en séries

\section{Le Merlin de la BBC (2008-2012)}

Entre détournement et réappropriation

\section{Aude Mairey}

\section{(2) OpenEdition}

\section{Journals}

Édition électronique

URL : https://journals.openedition.org/medievales/10797

DOI : $10.4000 /$ medievales. 10797

ISSN : 1777-5892

Éditeur

Presses universitaires de Vincennes

\section{Édition imprimée}

Date de publication : 20 août 2020

Pagination : $87-100$

ISBN : 978-2-37924-093-5

ISSN : 0751-2708

\section{Référence électronique}

Aude Mairey, «Le Merlin de la BBC (2008-2012) », Médiévales [En ligne], 78 | printemps 2020, mis en ligne le 02 janvier 2022, consulté le 22 avril 2022. URL : http://journals.openedition.org/medievales/ 10797 ; DOI : https://doi.org/10.4000/medievales.10797 


\title{
Aude Mairey
}

\section{Le Merlin de la BBC (2008-2012) \\ Entre détournement et réappropriation}

\begin{abstract}
Aucun jeune homme, quelle que soit sa valeur, ne peut connaître son destin. Il ne peut pas entrevoir le rôle qu'il jouera dans la grande histoire qui commence. [...] Comme tout un chacun, il doit apprendre jour après jour ; [...] et il en sera ainsi pour le jeune magicien qui arrive aux portes de Camelot. Un garçon, qui un jour, donnera naissance à une légende. Son nom ? Merlin ${ }^{1}$.
\end{abstract}

Entre 2008 et 2012, la BBC a diffusé cinq saisons d'une série sobrement intitulée Merlin, produite par la société Shine ; son succès a été notable, comme le suggèrent par exemple les sites de fans très complets tels que les «Wiki Merlin », en anglais et en français ${ }^{2}$. La première caractéristique de la série est la jeunesse de Merlin ; elle est revendiquée par ses créateurs, Julian Murphy et Johnny Capps, influencés notamment par la série américaine Smallville diffusée entre 2001 et 2010, qui retrace les premières années de Superman afin de surprendre d'emblée les spectateurs ${ }^{3}$. Merlin a donc le même âge qu'Arthur et le père de ce dernier, Uther Pendragon, est bien vivant. Le jeune sorcier devient le serviteur d'Arthur et ne peut s'essayer à l'apprentissage de ses pouvoirs que grâce au médecin du roi, Gaius, et

1. Merlin, S1/E1 : « No young man, no matter how great, can know his destiny. He cannot glimpse his part in the great story that is about to unfold [...]. We continue to follow the figure as he negotiates the final rocks that will lead him to the summit, as yet his face is still unseen [...]. Like everyone, he must live and learn [...]. The figure nears the brow. Ahead of him, slowly rising into view we see soaring spires and turrets... And so it will be for the young warlock arriving at the gates of Camelot. A boy that will in time father a legend. His name : Merlin. » Tous les scripts se trouvent sur le site [https://merlin.hypnoweb.net/merlin/], qui est une mine d'informations sur la série (résumés, personnages, casting, scènes coupées...). Les citations de la série, vérifiées, en sont issues.

2. Anglais : [https://merlin.fandom.com/wiki/Merlin]; français : [https://merlin. fandom.com/fr/wiki/Wiki_Merlin].

3. The Guardian, 29 août 2008, en ligne [https://www.theguardian.com/media/2008/ aug/29/bbc.television]. Une autre influence est celle du roman de M. STEWART, The Crystal Cave, New York, 1970. 
au dernier dragon retenu prisonnier par Uther, Kilgarrah. De même, les principaux personnages de la légende arthurienne qui apparaissent au fil des épisodes sont parfois bien loin des topoï traditionnels le plus souvent issus, pour le monde anglophone contemporain, de la Morte Arthur de Thomas Malory (1470) et de son adaptation moderne par Terence H. White, composée entre la fin des années 1930 et la fin des années $1950^{4}$. Guenièvre, par exemple, la plupart du temps appelée Gwen, est la servante de Morgane, elle-même pupille d'Uther. Par ailleurs, de nombreux épisodes comportent des moments cocasses, voire comiques, parfois répétitifs, mais assumés par les créateurs de la série qui visaient une audience variée et familiale («a three-generation show $\gg$ ).

De plus, l'espace même de la série - Albion, ancien nom mythique de la Grande-Bretagne ${ }^{5}$ - renvoie surtout à l'idée d'un espace lié à la fantasy, ce qui neutraliserait encore un peu plus les aspects «historiques» de la légende arthurienne, au moins dans les quatre premières saisons.

Pourtant, à y regarder de plus près, des enjeux politiques sous-jacents sont bien présents, tant dans la trame narrative de la série, en lien avec la tradition médiévale ou moderne, qu'en rapport avec des problématiques contemporaines concernant, notamment, la diversité et la tolérance. Mais ces deux éléments sont inextricablement liés dans la série et les échos entre sources médiévales et réécritures contemporaines doivent être reconnus. De ce fait, je me propose d'explorer certains aspects de cette série en lien avec les multiples archétypes du Merlin médiéval, pivot de ces échos, dont la malléabilité depuis les premières œuvres le mentionnant est reconnue de longue date ${ }^{6}$. En corollaire, je souhaite contribuer à une réflexion sur

4. Thomas Malory, The Works of Sir Thomas Malory, éd. E. VinaVer, rév. par P. J. C. FIELD, $3^{e}$ éd., Oxford, 1990 ; T. H. White, The Once and Future King, New York, 1958.

5. Dans l'Angleterre médiévale, le principal mythe fondateur implique un Troyen, Brutus, petit-fils d'Énée et qui a donné son nom à la Bretagne ; mais au XIV siècle, un mythe antérieur apparaît, bientôt relayé par la chronique la plus diffusée du Moyen Âge, le Brut, et donc bien connu : trente-trois filles d'un roi grec, menées par l'aînée Albina - d'où le nom d'Albion -, se seraient réfugiées dans les îles Britanniques après le meurtre de leurs trente-trois maris et y auraient conçu des enfants avec des géants, combattus par la suite par Brutus et ses compagnons. Voir J. P. CARLEY et J. CRICK, « Constructing Albion's Past : An Annotated Edition of De Origine Gigantum », dans J. P. CARLEY et F. RIDDY éd., Arthurian Literature, XIII, Cambridge, 1995, p. 41-114. Pour une présentation du Brut en français, voir A. MAIREY, « La tradition du Brut en moyen anglais à la fin du Moyen Âge », dans H. TÉTREL et G. Veysseyre éd., L'Historia regum Britannie de Geoffroy de Monmouth et les Bruts en Europe, t. 2 : Production, circulation et réception (XII'-XVI siècle), Paris, 2018, p. 169191. Notons toutefois que le nom «Albion » apparaît dès l'Antiquité, par ex. chez Pline : voir P. FreEmAn, «Albion, Albiones », dans J. T. Koch éd., Celtic Culture : A Historical Encyclopedia, Santa Barbara, 2006, vol. 1, p. 38-39.

6. Pour un panorama sur la question, voir par exemple S. KNIGHT, Merlin : Knowledge and Power through the Ages, Ithaca/Londres, 2009. 
la réception de la figure contemporaine de Merlin dont l'influence reste majeure (que l'on songe à Gandalf ou à Dumbledore, pour ne citer que les exemples les plus évidents), et ce d'autant plus que, dans les quelques études portant sur cette série, l'accent est plus souvent mis sur certains de ses thèmes majeurs - notamment la question de la place des femmes ou de la religion - que sur le protagoniste lui-même. Or, à mon sens, ces problématiques ne peuvent être étudiées que si l'on garde à l'esprit les interactions entre ces différents éléments.

Après avoir présenté la trame politique de la série et son appui sur des sources médiévales, je m'attacherai au portrait du jeune Merlin avant d'envisager les rapports entre magie, politique et religion ; je terminerai par la problématique de l'unité de la Grande-Bretagne telle qu'elle est abordée dans la série, en résonance avec les préoccupations actuelles la concernant.

\section{Une réécriture contemporaine ancrée dans les sources médiévales}

Les enjeux politiques qui constituent le fil rouge de la série apparaissent de manière inquiétante au jeune héros dès le tout premier épisode : envoyé à Camelot par sa mère, Hurith, aux bons soins de Gaius, le médecin du roi, par crainte que l'on ne découvre les capacités de son fils, Merlin assiste dès son arrivée à l'exécution d'un magicien et à la malédiction lancée par la mère de ce dernier sur Uther et, plus généralement, sur Camelot. La magie a en effet été bannie du royaume lors de la «Grande Purge » effectuée par le roi vingt ans plus tôt, ce qui constitue une déviation majeure par rapport à la plupart des récits médiévaux et post-médiévaux. En revanche, il n'est fait à aucun moment allusion au christianisme, contrairement à un grand nombre de réécritures contemporaines de la légende (la question ne se posant évidemment pas au Moyen Âge), telle l'œuvre de Marion Zimmer Bradley, dont l'assise repose sur l'opposition entre " ancienne religion » et christianisme ${ }^{7}$. De ce choix découle un discours politique dont l'interprétation est toutefois controversée. Selon David Tollerton, on observe « un souci récurrent de tolérance et de diversité tout au long de cette adaptation spécifique de la légende arthurienne pour le petit écran ${ }^{8} »$. Pour d'autres, telle Elysse Meredith, la série incarne au contraire une forme

7. M. Zimmer Bradley, The Mists of Avalon, New York, 1982. Pour une perspective plus générale sur la question du christianisme dans la matière arthurienne contemporaine, voir A. GAUTIER, Le Roi Arthur, Paris, 2019, p. 163-167, et W. BlANC, Le Roi Arthur. Un mythe contemporain, Paris, 2016, p. 335-336. Plus spécifiquement pour les romans historiques, voir M. Rolland, Le Roi Arthur. Un mythe hérö̈que au XXe siècle, Rennes, 2004.

8. D. C. TOLLERTON, « Multiculturalism, Diversity, and Religious Tolerance in Modern Britain and the BBC's Merlin », Arthuriana, 25/1 (2015), p. 113-127 (p. 113). Voir également W. BlANC, Le Roi Arthur..., p. 335-337. 
de conservatisme politique tendu vers l'unité de la Grande-Bretagne et la réaffirmation d'un pouvoir masculin'.

De plus, si, à l'instar de la structuration schématique de nombre de séries, certains épisodes portent sur une aventure ou une histoire spécifique et/ou anecdotique ${ }^{10}$, la progression de l'histoire de Merlin et d'Arthur suit son cours, inévitablement de plus en plus sombre. Elle mène à la disparition du roi et à l'espérance de son retour pour réunifier Albion ${ }^{11}$, même si cet effacement d'Arthur est lié au statut de la magie dans le royaume et non à la trahison familiale. Au fil du temps, Merlin apprend peu à peu à maîtriser ses pouvoirs, qu'il utilise parfois, à partir de la troisième saison, sous la forme d'un vieillard afin de ne pas se révéler à la cour d'Uther. Il prend alors le nom d'Emrys qui est son nom druidique dans la série ${ }^{12}$.

En apparence, donc, la série Merlin diffère d'adaptations plus traditionnelles, malgré, par exemple, la réintroduction du «vieux» Merlin par l'intermédiaire de sa transformation, mais aussi par l'imagemiroir que représente Gaius. Pourtant, au Moyen Âge, Merlin n'était pratiquement jamais représenté comme un vieillard, en tout cas sous sa véritable apparence ${ }^{13}$. C'est surtout au XIX ${ }^{e}$ et dans la première moitié du

9. E. T. MEREDITH, «Gendering Morals, Magic and Medievalism in the BBC's Merlin », dans M. PAGÈS et K. KINANE éd., The Middle Ages on Television. Critical Essays, Jefferson (NC), 2015, p. 158-173.

10. On peut bien sûr noter des parallèles avec les grands cycles médiévaux en prose médiévale, tel le Lancelot-Graal (XIII ${ }^{\mathrm{e}} \mathrm{s}$.), qui multiplient les épisodes anecdotiques et des digressions de tous ordres. Voir à ce sujet M. R. ELMES, «Episodic Arthur. Merlin, Camelot and the Visual Modernization of the Medieval Literary Romance Tradition », dans The Middle Ages on Television..., p.99-121 (p. 99) : «Les séries télévisées et les textes des romans médiévaux partagent plusieurs aspects supplémentaires : des épisodes individuels s'entrelacent pour former une unité narrative dominante ; un matériau original est adapté pour des publics différents ; des personnages sont introduits, transformés, combinés et omis pour convenir aux narrations variées; et de multiples écrivains sont impliqués dans leur élaboration. »

11. Autrement dit, il s'agit là d'un détournement de «l'espoir breton», exprimé dans certaines prophéties indiquant le possible retour du roi Arthur afin de sauver et de réunifier les peuples bretons. Voir V. GREENE, «Qui croit au retour d'Arthur?», Cahiers de civilisation médiévale, 42/4 (2002), p. 321-340.

12. L'équivalent latin de ce nom, Ambrosius, apparaît dans l'Historia Brittonum du pseudo-Nennius, composée dans le premier tiers du IX ${ }^{\mathrm{e}}$ siècle et qui relate un des épisodes majeurs de la légende de Merlin, repris et élaboré par Geoffroi de Monmouth : un enfant miraculeux prédit au roi Vortigern sa chute ainsi que les luttes entre Bretons et Saxons, symbolisées par la bataille de deux dragons enfouis sous les fondations de la forteresse que le roi essayait de construire sans succès (Nennius : British History and the Welsh Annals, éd. et trad. J. MORRIS, Londres et Chichester, 1980, p. 29-31 et p. 70-72). En ce qui concerne l'usage du nom en gallois, Eryri ou Emrys, son apparition n'est pas claire, même si une glose d'un des manuscrits de « Nennius » le mentionne ; de plus, ce nom est lié au site de Dinas Emrys (pays de Galles) qui aurait été le lieu de la construction de la forteresse.

13. Dans la Suite Vulgate de Merlin (années 1230), également connue sous le nom des Premiers Faits du roi Arthur, Merlin est considéré ainsi : «Et de fait il était rempli de 
$\mathrm{XX}^{\mathrm{e}}$ siècle qu'il est représenté ainsi. Or, comme l'a montré Jon Sherman ${ }^{14}$, l'écriture de la série révèle d'une part une maîtrise incontestée de sources médiévales majeures, bien au-delà de l'œuvre de Malory - à commencer par l'Histoire des rois de Bretagne rédigée dans la seconde moitié des années 1130 par Geoffroi de Monmouth, véritable «inventeur» de la matière arthurienne ${ }^{15}$, et d'autre part un tissu de références subtiles à ces sources (celtiques, anglaises, françaises), qui ne sont vraiment compréhensibles que par des spectateurs initiés à la légende arthurienne et à ses multiples variations. Le clin d'œil le plus spectaculaire en est sans doute l'introduction de Geoffroi lui-même comme personnage de la série - il est le bibliothécaire de la cour !

\section{Portrait en creux du jeune Merlin}

$\mathrm{Si}$, au Moyen Âge, la caractéristique la plus stable et la plus importante de Merlin est le don de prophétie, il incarne aussi d'autres archétypes maniés et remaniés au cours des siècles, des premiers poèmes brittoniques à Thomas Malory en passant par Geoffroi de Monmouth, (le pseudo-)Robert de Boron et le cycle du Lancelot-Graal - pour ne citer que les sources principales ${ }^{16}$. Il y est tour à tour, ou tout ensemble, barde et homme sauvage intimement lié à la nature, magicien né de l'union d'une femme et d'un incube, rédimé par Dieu (et, plus tard, lié au Graal), savant, conseiller et stratège militaire, ou encore amant victime de Viviane. La série de la BBC ne reprend pas l'ensemble de ces archétypes; et lorsque c'est le cas, ils sont souvent détournés. En outre, elle édulcore les aspects obscurs de la personnalité de Merlin (et, plus généralement, ceux d'autres personnages de la légende arthurienne tels que Lancelot et Guenièvre). Ceux qui sont employés transparaissent néanmoins de diverses manières et ont subi un

prouesse, fort et musclé, mais en même temps il était brun et maigre, et plus velu, comme un sauvage, que personne d'autre. Il était de noble origine du côté de sa mère, mais je ne vous dirai pas davantage ce qu'il en était du côté de son père » (Le Livre du Graal, vol. 1, éd. P. WALTER et al., Paris, 2001, p. 1205).

14. J. SHERMAN, « Source, Authority, and Audience in the BBC's Merlin », Arthuriana, 25/1 (2015), p. 82-100.

15. GeOFFroI DE Monmouth, Historia regum Britanniae, éd. N. Wright, Cambridge, 1985 ; ID., Histoire des rois de Bretagne, trad. L. MATHEY-MAILLE, Paris, 1993.

16. Pour une synthèse sur la création et les divers archétypes de Merlin, voir P. H. GoODRICH, «Introduction », dans P. H. GoODRICH et R. H. THOMPSON éd., Merlin : A Casebook, New York, 2003, p. 1-101. Selon certains auteurs, ces archétypes sont moins nombreux et peuvent varier : voir par exemple H. Fulton, « Arthur and Merlin in Early Welsh Literature: Fantasy and Magic Naturalism», dans EAD. éd., A Companion to Arthurian Literature, Oxford, 2009, p. 84-101. Je me permets de renvoyer à ma biographie sur Merlin, à paraître aux éditions Ellipses. 
profond remaniement, essentiel pour comprendre les enjeux politicoculturels de la série, qui doivent être analysés de manière nuancée.

Commençons par ce que notre héros n'est pas. Le jeune Merlin n'est pas le prophète par excellence, même s'il lui arrive d'avoir des visions. La plupart des nombreuses prophéties énoncées dans la série sont exprimées par d'autres personnages, notamment par le grand dragon, Kilgarrah ${ }^{17}$, mais aussi, par exemple, par Taliesin dans l'épisode 5 de la saison 3. Or, Taliesin, personnage à la fois historique (bien qu'il y ait des controverses sur ce point) et légendaire, est considéré comme un des plus grands bardes, et donc devins, du haut Moyen Âge ${ }^{18}$; il apparaît d'ailleurs dans un poème brittonique en conversation avec Myrddin (la forme gaélique de Merlin) ${ }^{19}$. Certes, il est souligné à plusieurs reprises que le libre arbitre peut changer le cours des événements, mais, en fin de compte, le destin d'Arthur et de Camelot est inévitable ${ }^{20}$.

Merlin n'apparaît jamais, non plus, comme un « homme sauvage ». Il a été élevé dans un village à la marge du royaume et vit dès le départ dans la forteresse de Camelot, qui ressemble surtout à un château de la fin du Moyen Âge ${ }^{21}$. Il n'est pas, non plus, un amant. À l'exception d'un léger flirt avec Gwen (Guenièvre) dans les premiers épisodes de la première saison, il vit une seule histoire d'amour le temps d'un épisode (S2/E9) avec une jeune druidesse, Freya, qui meurt d'une malédiction l'ayant transformée en bastet, une créature meurtrière ayant la forme d'une panthère dotée d'ailes de chauve-souris. Mais de manière générale, les histoires d'amour sont peu développées dans la série, à l'exception de celle d'Arthur et de Guenièvre, à peine troublée par Lancelot.

Pour d'autres caractéristiques, l'affaire est plus complexe, d'autant qu'il y a (heureusement...) des évolutions au long des cinq saisons de la

17. Sur ce dernier, voir infra.

18. Voir J. T. KOCH, « Taliesin (I). The Historical Taliesin », et M. HAYCOCK, « Taliesin (II). The Taliesin Tradition », dans J. T. KocH éd., Celtic Culture..., vol. 5, p. 1652-1653 et 1653-1656. Voir aussi P. WALTER, Merlin ou le Savoir du monde, Paris, 2000, p. 32-50, où Taliesin est présenté comme un « ancêtre de Merlin ».

19. «La conversation de Merlin et Taliesin » («Ymddiddan Myrddin a Thaliesin »), mis en forme probablement avant la fin du $\mathrm{XI}^{\mathrm{e}}$ siècle. Le poème est édité et traduit dans The Black Book of Carmarthen, introd. et trad. M. PENNAR, Lampeter, 1989.

20. Voir par exemple les paroles de Gaius après la vision par Merlin d'un futur effrayant dans un cristal magique : «C'était bien réel, mais ce n'était qu'une réalité. L'avenir n'est encore qu'un rêve qui n'existe pas. C'est nous qui le formons. C'est toi Merlin : les décisions que tu prendras, les actions qui seront les tiennes. Souviens-toi de cela. Allez, mange ta soupe avant qu'elle refroidisse. » (S2/E11 : «It was real, but it was just one reality. The future is as yet unshaped. It is we that shape it. It is you, Merlin. The decisions you make. The actions you take. Remember that. Eat your soup before it gets cold. »)

21. Et de fait, un des lieux de tournages de la série est le château de Pierrefonds, dans l'Oise, reconstruit presque totalement au début $\mathrm{du} \mathrm{XV}^{\mathrm{e}}$ siècle par Louis d'Orléans, puis à nouveau par Viollet-le-Duc au milieu du XIX ${ }^{\mathrm{e}}$ siècle. 
série. Durant les deux premières saisons, en particulier, Merlin n'apparaît pas dans son rôle de conseiller, ce qu'il est très clairement depuis Geoffroi de Monmouth, mais comme un «simple » serviteur. Nuançons toutefois : dès le premier épisode, il remet Arthur à sa place lors de leur première rencontre. Mais au fil des épisodes, ses conseils, formulés généralement de manière légère, au moins dans les trois premières saisons, sont de plus en plus nets, ce qu'Arthur finit d'ailleurs par reconnaître. Et finalement, à la fin de la saison 4 (E13), Merlin offre une nouvelle légitimité à Arthur en le conduisant à tirer Excalibur de son rocher grâce à son pouvoir de conviction... Néanmoins, l'ambivalence subsiste jusqu'à la toute fin de la série.

Par ailleurs, Merlin apparaît d'abord comme un «fils sans père », archétype développé pour la première fois dans l'Histoire des Bretons du pseudo-Nennius, sous le nom d'Ambrosius ${ }^{22}$ - c'est Geoffroi de Monmouth qui fait de Merlin le fils rédimé d'un incube ${ }^{23}$. Dans la série, il est envoyé par sa mère Hunith chez Gaius, on l'a vu, afin de le protéger et de lui enseigner ce dont il a besoin. Mais ce dernier est en fait le frère d'Hunith et donc l'oncle maternel de Merlin ; il est par conséquent tout à fait légitime pour répondre à la demande de sa sœur :

Il a besoin d'une main qu'il puisse tenir, d'une voix qui le guide, de quelqu'un qui l'aide à trouver un usage de ses dons. Je vous supplie, si vous comprenez l'amour qu'une mère porte à son fils, de le garder à l'abri du danger. Et que Dieu vous protège tous les deux ${ }^{24}$.

Gaius a donc d'emblée un rôle paternel et éducatif dont il ne se départira jamais, ce qui peut renvoyer, au passage, à l'importance des oncles maternels dans les sociétés médiévales. De plus, il a besoin d'apprendre, contrairement à la grande majorité des récits merliniens où il est au contraire celui qui enseigne - à Uther puis à Arthur en particulier; c'est d'ailleurs bien ce qui a fait le succès de la première partie de l'œuvre de Terence White adaptée par Disney.

Mais il s'avère que, finalement, Merlin a bien un père que sa mère ne voulait pas révéler : il s'agit de Balinor, le dernier dragonnier qui a échappé à la Grande Purge et qui se révèle à la fin de la saison 2 pour sauver Camelot après la libération du vieux dragon par Merlin. Ce dernier n'est donc pas le « fils sans père » médiéval, mais celui d'une figure contemporaine de la

22. Voir supra, n. 13 .

23. GEOFFROI DE MONMOUTH, Histoire des rois de Bretagne, chap. 107, p. 155-156.

24. S1/E1 : «In these times, he needs a hand to hold, a voice to guide, someone that might help him find a purpose for his gifts. To this end, I hope and trust, my dear brother, that you will have it in your heart to be that person. I beg you, if you understand a mother's love for her son, keep him safe. » 
fantasy, même si son affinité avec les dragons est un aspect important du personnage dès le Moyen Âge, nous y reviendrons ${ }^{25}$.

\section{Merlin, la magie et I' « Ancienne religion »}

Venons-en à l'archétype le plus important dans la série, celui du magicien. Merlin incarne d'abord, comme d'autres personnages, une magie «naturelle », innée, qu'il doit simplement apprendre à contrôler. Mais certains aspects de cette magie, telle qu'elle pouvait être définie au Moyen Âge - avec toutefois des évolutions majeures à partir du XIII ${ }^{\mathrm{e}}$ siècle en lien avec l'introduction de la philosophie naturelle aristotélicienne ${ }^{26}$ peuvent également être appris. On y retrouve en effet l'importance de la maîtrise des éléments, du pouvoir des plantes et des pierres, le tout en lien avec l'alchimie et la médecine ${ }^{27}$. Gaius en est le représentant et va s'appliquer à enseigner son savoir à Merlin, même s'il évoque parfois une distinction anachronique entre science et magie. Dans l'épisode 3 de la saison 1, par exemple, alors qu'une épidémie répandue par Nimueh ${ }^{28}$ menace les habitants de Camelot, il affirme à Arthur, venu fouiller chez lui pour trouver des preuves d'agissements magiques, que sa vie est consacrée à la compréhension de la science. Gaius est toutefois tiraillé car il est en réalité un magicien, abstinent depuis la Grande Purge : de fait, il donne à Merlin son livre de magie dès la fin du tout premier épisode et finit par pratiquer à nouveau la magie lorsque cela devient nécessaire. Quant à la magie noire, parfois qualifiée de nécromancie ou nigromancie mais qui n'était pas toujours considérée comme dangereuse au Moyen Âge, elle présente également un mélange de dons innés et d'apprentissage, comme c'est le cas pour Morgane ou d'autres sorciers malveillants dans la série, tel Cornelius Sigan dans le premier épisode de la saison $2^{29}$.

25. Voir M. BosT-FIEVET, « Dragons », dans A. Besson éd., Dictionnaire de la fantasy, Paris, 2018, p. 94-101.

26. Voir C. SAunders, «Religion and Magic », dans E. ARChIBALD et A. PutTER éd., The Cambridge Companion to Arthurian Legend, Cambridge, 2009, p. 201-217 ; H. Fulton, «Arthur and Merlin... »; J.-P. BOUDET, Entre science et nigromance. Astrologie, divination et magie dans l'Occident médiéval (XII'-XVe siècle), Paris, 2006.

27. Le mélange d'inné et d'acquis se retrouve par exemple dans les transformations de Merlin à partir de la saison 3 : il doit d'abord user de potions pour se métamorphoser (comme chez Geoffroi de Monmouth), mais n'en a plus besoin à la fin, lorsque l'étendue de ses pouvoirs lui est révélée, dans l'avant-dernier épisode de la saison 5.

28. C'est à l'origine, surtout dans les îles Britanniques, un des nombreux noms de la Dame du Lac, plus connue en France sous le nom de Viviane.

29. Sur la nécromancie ou nigromancie, au Moyen Âge, voir par ex. J.-P. BouDET, Entre science et nigromance..., particulièrement p. $125 \mathrm{sq}$. 
La magie de Merlin est aussi associée, parfois, à l'«Ancienne religion » et aux druides, pratiquement éradiqués par la Purge ${ }^{30}$. Or, de nombreux magiciens (et magiciennes) ne sont devenus hostiles qu'à la suite de cette Purge qui a terrassé une grande partie des leurs, d'autant qu'elle a été lancée par Uther pour des raisons personnelles : sa femme Ygerne étant stérile, il avait demandé à Nimueh d'y remédier. Arthur est né mais Ygerne est morte en couches - une vie pour une vie. Dès lors, Uther incarne l'intolérance envers toute forme de magie. Arthur n'apprend la vérité qu'à l'épisode 8 de la saison 2 , mais auparavant il incite plusieurs fois son père à la modération, par exemple lorsqu'il lui demande de ne pas exécuter un druide et son fils, Mordred en l'occurrence (S1/E8). Néanmoins, comme la magie n'a pu sauver son père, Arthur n'autorise pas son usage une fois devenu roi, au début de la saison 4, au grand désespoir de Merlin. Quant à Morgane, c'est sa haine croissante de la tyrannie d'Uther qui la conduit vers les chemins obscurs...

Selon Elysse Meredith, pourtant, si les druides sont dans l'ensemble des personnages pacifiques qui n'ont recours à la violence qu'en réponse à la répression d'Uther, l'organisation même de l'Ancienne religion est malveillante et ses prêtresses - Nimueh, Morgause ${ }^{31}$, puis Morgane incarnent la violence d'un système matriarcal destructeur. Meredith note toutefois que, si leur vilenie est inéluctable, elle s'est en grande partie construite en réaction aux actes des hommes ${ }^{32}$. D'ailleurs, le jeune druide Mordred n'apparaît pas d'emblée comme un être malveillant et devient même chevalier de la Table ronde, avant de se ranger aux côtés de Morgane suite aux «trahisons » d'Arthur et de Merlin (S5/E11).

Cela dit, il est souvent rappelé que la magie n'est, en elle-même, ni bonne ni mauvaise mais qu'elle n'est qu'un outil : Gaius lui-même l'affirme dans l'épisode 3 de la première saison. De fait, la série offre, me semble-t-il, une image complexe et non manichéenne de la magie, dont les héros positifs tentent de restaurer une harmonie naturelle et de réenchanter le monde - ce qui, selon William Blanc par exemple, constitue un aspect important de nombreuses œuvres récentes de la fantasy, et particulièrement celles qui concernent Merlin ${ }^{33}$. Mais cette présentation de la magie renvoie également, bien sûr, à des problématiques contemporaines beaucoup plus vastes, concernant la perception et la représentation des outils technologiques ou des avancées scientifiques; elle s'inscrit donc dans un large contexte

30. Ce thème de l'ancienne religion apparaît notamment dans l'œuvre de Marion Zimmer Bradley, mais en opposition à l'absence de religion plutôt qu'au christianisme (voir supra).

31. Dans certains récits médiévaux, Morgause est une des tantes d'Arthur, celle avec laquelle il commet le péché d'inceste qui engendre Mordred.

32. E. T. MEREDITH, « Gendering Morals... ».

33. W. BlanC, Le Roi Arthur..., p. 332-336. 
culturel marqué par les préoccupations autour des usages des sciences et des techniques, que ce soit au Moyen Âge ou de nos jours.

Cette volonté de marquer la magie comme un ensemble de techniques pouvant être utilisées pour le bien comme pour le mal se retrouve en partie dans la relation du magicien avec les dragons. Comme l'a montré Philippe Walter, cette relation est ancienne ${ }^{34}$. Dans les principaux épisodes récurrents des récits médiévaux, le dragon est un animal symbolique majeur. C'est le cas, par exemple, lorsque Uther aperçoit un dragon dans le ciel lors de la mort de son frère Ambrosius et que Merlin lui explique qu'il représente son avènement ; c'est d'ailleurs à ce moment qu'Uther prend le titre de Pendragon ${ }^{35}$. De même, Merlin conduit parfois l'armée, notamment dans des romans français du XIII ${ }^{\mathrm{e}}$ siècle, en brandissant une bannière ornée d'un dragon qui crache du feu pour effrayer les ennemis ${ }^{36}$. Les dragons sont donc associés de manière positive à Merlin. Dans la série, le dragon Kilgarrah, dernier de son espèce enfermé par Uther lors de la Purge, est cependant un être plus complexe. Dès le premier épisode, il appelle Merlin et va devenir son second conseiller, même s'il n'est pas souvent écouté. Et lorsque le dragon lui explique sa destinée, il ne tranche pas entre le bien et le mal :

Kilgarrah : Oui. Arthur est le roi présent et à venir qui va unifier la terre d'Albion.

Merlin : D'accord...

Kilgarrah : Mais il est confronté à de nombreuses menaces d'amis autant que d'ennemis.

Merlin : Je ne vois pas ce que ça a à voir avec moi.

Kilgarrah : Ça a tout à voir, car sans toi Arthur ne réussira jamais. Et sans toi, il n'y aura pas d'Albion.

Merlin : Non ! Ce que vous dites est faux !

Kilgarrah : Il n'y a pas de vrai ni de faux, seulement ce qui existe et ce qui n'existe pas.

Merlin : Mais je suis sérieux : s'il y a des gens qui veulent le tuer, ils peuvent le faire ! Je suis prêt à les aider.

Kilgarrah (riant) : Aucun de nous ne peut choisir son destin, Merlin. Et aucun de nous ne peut y échapper.

Merlin : Non... Pas question, non ! Non. Il doit y avoir un autre Arthur parce que celui-là est un crétin.

Kilgarrah : Peut-être est-ce ton destin que de changer ce détail ${ }^{37}$ ?

34. P. WALTER, Merlin..., p. 129-145.

35. GeOFFroI DE Monmouth, Histoire des rois de Bretagne, chap. 133, p. 192-193. 909.

36. Voir par exemple Les Premiers Faits du roi Arthur, dans Le Livre du Graal..., p. 908-

37. S1/E1 : «Kilgarrah : Arthur is the once and future king who will unite the land of Albion. / Merlin : I don't see what that has to do with me. / Kilgarrah : Everything. Without 
En outre, une fois qu'il a été libéré par Merlin à la fin de la saison 2, le premier acte de Kilgarrah est de ravager Camelot, jusqu'à ce qu'il soit arrêté par le jeune magicien devenu le dernier dragonnier après la mort de son père. Dans les saisons suivantes, il devient néanmoins son ami, moins narquois et plus bienveillant (même s'il est obligé de lui obéir). Il est d'abord préoccupé par le sort d'Arthur et d'Albion.

Cependant, dans la saison 4 (E4), Kilgarrah se trompe sur un point essentiel : alors qu'un magicien a réussi à retrouver un œuf de dragon vieux de plusieurs siècles, c'est Merlin, le seul à en avoir le pouvoir, qui le fait éclore. En sort un dragon blanc qu'il nomme Athusa (rayon de soleil). Selon Kilgarrah, c'est un heureux présage. Mais quelques épisodes plus tard, Athusa sauve Morgane d'une mort certaine et devient sa «chose », bientôt difforme, qui l'aidera dans son combat contre Arthur. Or, chez le pseudoNennius, repris par Geoffroi de Monmouth, le dragon rouge est le symbole des Bretons et le dragon blanc celui des Saxons auxquels Morgane s'allie dans la dernière saison afin de faire tomber Arthur et, par là même, Albion ${ }^{38}$.

\section{Le rêve brisé de l'unité}

Albion, l'espace qui doit être unifié par Arthur (et dont Merlin est le garant) est certes, on l'a vu, le premier nom mythique de la GrandeBretagne, précédant la fondation de cette dernière par Brutus et les Troyens ${ }^{39}$. Cela apparaît d'ailleurs subtilement dans le dernier épisode de la saison 4, lorsque Merlin raconte la légende d'Excalibur à Arthur ; il attribue l'existence de l'épée à... Bruta :

Merlin: Bien des années avant nous, bien avant l'apparition des cinq royaumes, cette terre n'était que chaos, carnage et guerres. Mais un jour, un homme décida de mettre un terme à ce cycle sans fin. Il réunit tous les anciens de chaque tribu pour diviser équitablement le territoire. Chacun respecterait les frontières et gouvernerait comme il l'entendrait. Le premier

you, Arthur will never succeed. Without you, there will be no Albion. / Merlin : No... No, you've got this wrong. / Kilgarrah : There is no right or wrong. Only what is and what isn't. / Merlin : I'm serious... If anyone wants to kill him they can go ahead. In fact, I'll give them a hand. / Kilgarrah (amused) : None of us choose our destiny, Merlin... (then with a hint of sadness) And none of us can escape it. / Merlin : No, no way, there must be some other Arthur, because this one's a moron. / Kilgarrah : Perhaps it's your destiny to change that. »

38. Nennius : British History..., chap. 42, p. 71 ; GEOFFROI DE MONMOUTH, Histoire des rois de Bretagne, chap. 111, p. 158-159.

39. La matière troyenne et la matière arthurienne sont deux piliers des justifications politiques dans les îles Britanniques tout au long du Moyen Âge - Geoffroi de Monmouth en étant une fois de plus le précurseur. Il est donc particulièrement intéressant que les auteurs aient décidé de fondre ces deux matières à un des moments les plus cruciaux de la série. 
roi de Camelot était, lui, l'ancêtre de tous ceux qui ont suivi, le vôtre aussi Arthur.

Arthur : Oui, Bruta.

$[\ldots]$

Merlin : Quand Bruta fut sur son lit de mort, il demanda à être conduit au fin fond de la forêt. Et là, avec ses dernières forces, il enfonça son épée dans un rocher. Si sa descendance était remise en question, cela serait une épreuve décisive, car seul un authentique Roi de Camelot pourrait la libérer ${ }^{40}$.

Toutefois, contrairement à ce qui se passe dans les récits médiévaux, Arthur ne parvient pas à unifier la Bretagne/Albion avant sa disparition. Selon certains, c'est un signe de conservatisme et d'anxiété lié aux problématiques contemporaines concernant l'unité du Royaume-Uni. Pour Dragos Manea, par exemple, « Merlin met en avant sa propre (re)configuration de l'identité nationale britannique qui, de manière très semblable à ses prédécesseurs de la fin du Moyen Âge, considère l'anglicité comme une caractéristique déterminante $^{41} \gg$. La critique d'Elysse Meredith est plus sévère encore :

La série [...] nous laisse gouvernés par la gentille, passive, sage et séculière Guenièvre qui rappelle l'actuelle reine britannique. [...] Merlin attend toujours le retour d'Arthur : un gouverneur mâle, un unificateur politique et un conquérant, une autorité séculière et un héros légendaire. Il attend la personne qui résoudra les préoccupations sociales de la série et « fera surgir le monde dont nous rêvons $»^{42}$.

Je ne pense pas que l'attente par Merlin du retour d'Arthur (dernière scène de la série, dans le monde actuel) implique forcément l'attente d'un conquérant viril. L'espoir du retour d'Arthur et de l'unification définitive de la Bretagne contre les Saxons (ses principaux ennemis dans toutes les versions médiévales de la matière arthurienne), qui apparaissent en force dans la dernière saison où ils sont alliés à Morgane, suggère que la série n'est pas un acte de simple «propagande». Comme chaque adaptation,

40. S4/E13 : «Merlin : Many years ago, before the birth of the five kingdoms, this land was in an endless cycle of bloodshed and war, but one man was determined to end all that. $\mathrm{He}$ gathered together the elders of each tribe and drew up plans for the lands to be divided. Each would respect the others' boundaries, and each would rule over the land as they saw fit. That man was Camelot's first king, ancestor to all that followed, including you, Arthur. / Arthur : Bruta. [...] / Merlin : When Bruta was on his deathbed, he asked to be taken deep into the forest. There, with the last of his strength, he thrust his sword into a rock. If his lineage was ever questioned, this would form a test. Only a true king of Camelot could pull the weapon free. »

41. D. ManeA, «The Shadow Upon the Screen : Merlin (2008-2012) and the Matter of Britain », University of Bucharest Review, $2^{\mathrm{e}}$ série, $2 / 3$ (2013), p. $53-61$ (p. 60).

42. E. T. MEREDITH, « Gendering Morals... », p. 167-168. 
comme chaque translatio, cette série repose la question plus large de l'identité «britannique » dans ses différentes composantes. Elle aborde aussi, on l'a vu, les anxiétés de nos sociétés post-industrielles, mais pas forcément de manière réactionnaire. Comme l'a souligné William Blanc, que je rejoins sur ce point, la présence croissante de Merlin ces dernières décennies « répond à l'angoisse de la modernité confrontée à sa propre autodestruction $^{43} »$.

En fin de compte, cette série - dont l'objectif est avant tout de divertir le grand public auquel elle est destinée - présente pourtant des enjeux politiques et culturels qui interrogent tant notre imaginaire que nos représentations contemporaines, en faisant usage de caractéristiques présentes dans la matière arthurienne aussi bien médiévale que moderne. La manière dont sont traités tant la magie que l'Ancienne religion centrée sur le matriarcat, ou encore le rêve déçu de l'unification d'Albion, apparaît comme autant d'éléments de réflexion sur la nature du pouvoir et la diversité de nos sociétés.

Le Merlin de la BBC constitue donc un exemple de la perpétuelle capacité de renouvellement de ce personnage mythique (et plus généralement de la matière arthurienne), non seulement à des fins divertissantes, mais aussi politiques (au sens le plus large du terme). En cela, elle rejoint de nombreuses séries contemporaines qui, quel que soit le sujet abordé, interrogent notre monde d'ici et maintenant. Mais elle conserve aussi, à sa manière, des éléments essentiels qui ont assuré la popularité presque continue de Merlin depuis le Moyen Âge : un magicien métamorphe et rieur, mais loyal jusqu'à la fin des temps.

Aude Mairey - LaMOP - CNRS/Université Paris 1

Le Merlin de la BBC (2008-2012). Entre détournement et réappropriation

Entre 2008 et 2012, la BBC a diffusé une série tous publics intitulée Merlin, qui a détourné nombre de topoï de la matière arthurienne. Merlin est jeune et serviteur du prince Arthur, Uther est vivant et la magie est interdite dans son royaume de Camelot. Si plusieurs études ont analysé quelques grands thèmes de la série - la tolérance, la place des femmes... -, Merlin lui-même en a parfois été oublié. Cet article se propose d'explorer ce personnage au regard de son riche passé légendaire et de contribuer à la réflexion sur sa réception contemporaine, tout en tenant compte des enjeux politiques, bien présents mais controversés.

arts visuels, matière arthurienne, médiévalisme, Merlin, séries télévisées, télévision.

43. W. Blanc, Le Roi Arthur..., p. 338. 


\section{The BBC's Merlin. Between Distortion and Appropriation}

The BBC's TV series Merlin was broadcast between 2008 and 2012. This "three-generation" series twisted many topoi drawn from Arthurian material: Merlin is young and a servant of Prince Arthur, while Uther is alive and has banned magic from his kingdom of Camelot. Several studies have analyzed some major themes of the series-including tolerance and the status of women-, but Merlin himself has not been really investigated as its principal character. In this paper, I will study him in light of his rich legendary past, aiming to contribute to the reflection on his contemporary reception while taking into account the immediate, often controversial, political issues of the series.

Arthurian material, medievalism, Merlin, television, TV series, visual culture. 